\title{
Population-based BRCA1/BRCA2 screening in Ashkenazi Jews: A call for evidence
}

\author{
Ephrat Levy-Lahad, MD
}

$T$ he promise of the genetic revolution is often encapsulated by the term "personalized medicine," but many of its benefits are expected to be in the realm of public health and prevention, through population genetic screening of asymptomatic individuals. As noted by Rubinstein et al. ${ }^{1}$ in this issue, $B R C A 1 / B R C A 2$ testing in the Ashkenazi Jewish population, aimed at reducing morbidity and mortality of breast/ovarian cancer, is seemingly an obvious candidate for such a screening program. In Ashkenazi Jews, three common, easily tested mutations account for the majority of deleterious alleles, effective preventive measures for $B R C A 1 / B R C A 2$ carriers exist, and this ethnic group has historically embraced genetic testing, albeit carrier screening for recessive disorders. However, when measured against accepted criteria for population screening, ${ }^{2}$ significant gaps remain to be addressed before such a program is initiated.

Proposed principles for genetic susceptibility screening include three stages: (1) public health assessment, i.e., establishing the burden and prevalence of the disease and its natural history, including that of the latent stage (i.e., penetrance), (2) evaluation of the test and interventions for those testing positive, and (3) policy development and screening implementation, including availability of adequate laboratory and clinical facilities and determination of cost effectiveness. ${ }^{2}$ These roughly parallel the first three stages (T1-T3) of the translational framework proposed for genomic medicine, ${ }^{3}$ which also includes an additional stage (T4) in which the "real-world" effects of a test are evaluated. Although establishing these stages sequentially would lead to a solid evidence base, there is also concern that such an orthodox approach may delay delivery of important scientific advances to an increasingly impatient public, awaiting the fruits of the genomic era. ${ }^{4}$ Although the latter stages of implementation, dissemination and economic reassessment may be amenable to a reiterative process, defining the optimal threshold of clinical utility (Stages 1-2), remains a critical gateway to the entire process. ${ }^{3,4}$

In the case of $B R C A 1 / B R C A 2$ testing, one missing piece of information is the cancer risk for carriers identified in the population, irrespective of personal or family history of cancer. In 2005, the US Preventive Services Task Force recommended $B R C A 1 / B R C A 2$ testing only for women with an increased-risk family history, defined in Ashkenazi Jews as any first-degree relative (or two second-degree relatives

From the Medical Genetics Institute, Shaare Zedek Medical Center, Jerusalem, Israel; and Department of Genetics, Hebrew University Medical School, Jerusalem, Israel.

Ephrat Levy-Lahad, MD, Medical Genetics Institute, Shaare Zedek Medical Center, PO Box 3235, Jerusalem 91031, Israel. E-mail: lahad@szmc.org.il.

Disclosure: The author declares no conflict of interest

Submitted for publication July 11, 2009

Accepted for publication July 14, 2009.

Published online ahead of print August 17, 2009.

DOI: $10.1097 /$ GIM.0b013e3181b765d3 on the same side of the family) with breast or ovarian cancer. ${ }^{5}$ As noted by Rubinstein et al., ${ }^{1}$ subsequent studies have shown that approximately half of $B R C A 1 / B R C A 2$ carriers identified after diagnosis of breast cancer and one third of carriers identified after diagnosis of ovarian cancer do not have significant family history of either cancer.

This has been interpreted to mean that close to half of carriers at risk for cancer would not be identified by family history or cascade approaches. However, lack of family history often reflects small family size, and therefore fewer individual women at potential risk. More importantly, data on the proportion of affected carriers lacking family history are based on women who already developed cancer, who may harbor additional risk factors, and who are not necessarily representative of all carriers in the population. ${ }^{6}$ Theoretically, there could be individuals or families with much lower penetrance of $B R C A 1 / B R C A 2$ mutations who would not be identified through affected women, and this would have obvious implications for population screening. Indeed, the lowest penetrance estimates quoted ${ }^{1}$ are derived from the single population-based study performed in Ashkenazi Jews.

In this respect, the history of testing for hereditary hemochromatosis $(\mathrm{HH})$ is a cautionary example of the importance of population-based penetrance estimates. ${ }^{7}$ Universal screening for $\mathrm{HH}$ was seriously discussed because almost all people clinically affected with $\mathrm{HH}$ are homozygous for the HFE C282Y mutation, homozygosity for this mutation is common $(\sim 0.5 \%$ in Northwest Europeans) and a simple, inexpensive preventive measure (phlebotomy) exists. However, despite high rates of biochemical penetrance (iron overload), large population-based studies determined that $<5 \%$ of $\mathrm{C} 282 \mathrm{Y}$ homozygotes develop cirrhosis, the clinically significant outcome of $\mathrm{HH}$.

Research addressing Stages 1-2 led to evidence-based recommendations against population-based screening of HFE mutations (reviewed in Ref. 7). Multiple lines of evidence suggest that population-level penetrance of $B R C A 1 / B R C A 2$ mutations will not be low, but since this can be feasibly determined in the Ashkenazi population it should be investigated, just as $\mathrm{HH}$ was evaluated in the European population. In Israel, such studies are underway, with the specific aim of assessing the evidence for $B R C A 1 / B R C A 2$ screening.

Additional evidence gaps are present in the next, Stages 3-4, steps of a translational screening program. As discussed by Rubinstein et al., ${ }^{1}$ we have yet to determine the most effective pretest and post-test processes. Pretesting traditional genetic counseling should be compared with other methods, which may be less costly. ${ }^{8}$ It is unclear if all screened individuals should undergo an identical process, or whether they should be stratified based on family history and other risk factors. In the post-test process, such stratification may be important to avoid false reassurance in women who remain at risk even following a negative screen.

Rubinstein et al. ${ }^{1}$ have specifically attempted to address the cost effectiveness of a BRCA1/BRCA2 screening program. However, in a rapidly evolving field, comprehensive economic analysis is fraught with difficulty. The recent introduction of breast magnetic resonance imaging surveillance has been highly 
variable in terms of access and insurance coverage, and data on uptake are limited. Thus, while in the "real world," a woman identified as a carrier learns that her risk for breast cancer is higher than that for ovarian cancer, with immediate implications for costs and benefits of breast cancer surveillance and prevention, the cost effectiveness of $B R C A 1 / B R C A 2$ screening for breast cancer outcomes was not assessed in this study. ${ }^{1}$ This should be tempered by the observation that although economic analyses are considered integral to the appraisal of screening programs, relatively few preventive measures are cost saving, ${ }^{9}$ and historically cost effectiveness has not played a major role in adoption of genetic screening tests. ${ }^{7}$

How do we proceed? Rubinstein et al. ${ }^{1}$ note that in the Tay Sachs screening program, much of the information on mutation prevalence, penetrance, and test performance was determined by first establishing a screening program, and only subsequently collecting the data as it accrued. They suggest that following community and professional dialogue, a similar approach should be taken with $B R C A 1 / B R C A 2$ screening, because without programmatic screening, $B R C A 1 / B R C A 2$ testing in healthy Ashkenazi women without family history will occur spontaneously, in a manner that will hamper any possibility of future assessment. Although Tay Sachs testing is undoubtedly a major success of genetic screening, in Ashkenazi Jews the legacy of the programmatic process has also contributed to the rapid adoption of carrier screening for practically any disease for which testing is possible (e.g., connexin 26-associated deafness and alpha-1 antitrypsin). Debatable consequences of this approach include pregnancy terminations in cases of low penetrant, treatable conditions, such as Gaucher disease. ${ }^{10}$ It could be argued that rather than starting screening ad hoc, the very dialogue proposed can and should lead to well-designed studies that address the numerous issues associated with BRCA1/ $B R C A 2$ screening in the Ashkenazi population. Such studies are feasible precisely because of the genetic and social architecture of Ashkenazi Jews, and with the advent of cheaper sequencing technologies, they will be applicable in the near future to women of all ancestries.

\section{ACKNOWLEDGMENTS}

The author's work is funded by the Breast Cancer Research Foundation and the Israel Cancer Association.

\section{REFERENCES}

1. Rubinstein W, Jiang H, Dellefave L, Rademaker AW. Cost-effectiveness of population-based $B R C A 1 / 2$ testing and ovarian cancer prevention for Ashkenazi Jews: a call for dialogue. Genet Med 2009;11:629-639.

2. Khoury MJ, McCabe LL, McCabe ER. Population screening in the age of genomic medicine. $N$ Engl J Med 2003;348:1604-1605.

3. Khoury MJ, Gwinn M, Yoon PW, Dowling N, Moore CA, Bradley L. The continuum of translation research in genomic medicine: how can we accelerate the appropriate integration of human genome discoveries into health care and disease prevention? Genet Med 2007;9:665-674.

4. Khoury MJ, Berg A, Coates R, Evans J, Teutsch SM, Bradley LA. The evidence dilemma in genomic medicine. Health Aff (Millwood) 2008;27: 1600-1611.

5. U.S. Preventive Services Task Force. Genetic risk assessment and BRCA mutation testing for breast and ovarian cancer susceptibility: recommendation statement. Ann Intern Med 2005;143:355-361.

6. Begg CB. On the use of familial aggregation in population-based case probands for calculating penetrance. J Natl Cancer Inst 2002;94:1221-1226.

7. Grosse SD, Rogowski WH, Ross LF, Cornel MC, Dondorp WJ, Khoury MJ. Population screening for genetic disorders in the 21 st century: evidence, economics, and ethics [published online ahead of print June 29, 2009]. Public Health Genomics.

8. Calzone KA, Prindiville SA, Jourkiv O, et al. Randomized comparison of group versus individual genetic education and counseling for familial breast and/or ovarian cancer. J Clin Oncol 2005;23:3455-3464.

9. Cohen JT, Neumann PJ, Weinstein MC. Does preventive care save money? Health economics and the presidential candidates. $N$ Engl J Med 2008;358: 661-663.

10. Beutler E. Carrier screening for Gaucher disease: more harm than good? JAMA 2007;298:1329-1331. 